\title{
Robin's criterion on divisibility
}

\author{
Frank Vega
}

the date of receipt and acceptance should be inserted later

\begin{abstract}
Robin's criterion states that the Riemann hypothesis is true if and only if the inequality $\sigma(n)<e^{\gamma} \times n \times \log \log n$ holds for all natural numbers $n>5040$, where $\sigma(n)$ is the sum-of-divisors function of $n$ and $\gamma \approx 0.57721$ is the Euler-Mascheroni constant. We show that the Robin inequality is true for all natural numbers $n>5040$ that are not divisible by some prime between 2 and 1771559 . We prove that the Robin inequality holds when $\frac{\pi^{2}}{6} \times \log \log n^{\prime} \leq \log \log n$ for some $n>5040$ where $n^{\prime}$ is the square free kernel of the natural number $n$. The possible smallest counterexample $n>$ 5040 of the Robin inequality implies that $q_{m}>e^{30.99733785}, 1<\frac{1.25 \times \log (4.7312714399)}{\log q_{m}}+$ $\frac{\log N_{m}}{\log n},(\log n)^{\beta}<1.0501395952 \times \log \left(N_{m}\right)$ and $n<(4.7312714399)^{m} \times N_{m}$, where $N_{m}=\prod_{i=1}^{m} q_{i}$ is the primorial number of order $m, q_{m}$ is the largest prime divisor of $n$ and $\beta=\prod_{i=1}^{m} \frac{q_{i}^{a_{i}+1}}{q_{i}^{a_{i}+1}-1}$ when $n$ is an Hardy-Ramanujan integer of the form $\prod_{i=1}^{m} q_{i}^{a_{i}}$.
\end{abstract}

Keywords Riemann hypothesis - Robin inequality - sum-of-divisors function · prime numbers $\cdot$ Riemann zeta function

Mathematics Subject Classification (2010) MSC 11M26 · MSC 11A41 · MSC $11 \mathrm{~A} 25$

\section{Introduction}

In mathematics, the Riemann hypothesis is a conjecture that the Riemann zeta function has its zeros only at the negative even integers and complex numbers with real part $\frac{1}{2}$ [3]. As usual $\sigma(n)$ is the sum-of-divisors function of $n$ [4]:

$$
\sum_{d \mid n} d
$$

F. Vega

CopSonic, 1471 Route de Saint-Nauphary 82000 Montauban, France

ORCiD: 0000-0001-8210-4126

E-mail: vega.frank@gmail.com 
where $d \mid n$ means the integer $d$ divides $n$ and $d \nmid n$ means the integer $d$ does not divide $n$. Define $f(n)$ to be $\frac{\sigma(n)}{n}$. Say Robins $(n)$ holds provided

$$
f(n)<e^{\gamma} \times \log \log n .
$$

The constant $\gamma \approx 0.57721$ is the Euler-Mascheroni constant and log is the natural logarithm. The importance of this property is:

Theorem 1.1 Robins $(n)$ holds for all natural numbers $n>5040$ if and only if the Riemann hypothesis is true [8].

It is known that Robins $(n)$ holds for many classes of numbers $n$. Robins $(n)$ holds for all natural numbers $n>5040$ that are not divisible by 2 [4]. On the one hand, we prove that Robins $(n)$ holds for all natural numbers $n>5040$ that are not divisible by some prime between 3 and 1771559 . We recall that an integer $n$ is said to be square free if for every prime divisor $q$ of $n$ we have $q^{2} \nmid n$ [4].

Theorem 1.2 Robins $(n)$ holds for all natural numbers $n>5040$ that are square free [4].

In addition, we show that Robins $(n)$ holds for some $n>5040$ when $\frac{\pi^{2}}{6} \times \log \log n^{\prime} \leq$ $\log \log n$ such that $n^{\prime}$ is the square free kernel of the natural number $n$. Let $q_{1}=2, q_{2}=$ $3, \ldots, q_{m}$ denote the first $m$ consecutive primes, then an integer of the form $\prod_{i=1}^{m} q_{i}^{a_{i}}$ with $a_{1} \geq a_{2} \geq \cdots \geq a_{m} \geq 0$ is called an Hardy-Ramanujan integer [4]. A natural number $n$ is called superabundant precisely when, for all natural numbers $m<n$

$$
f(m)<f(n) .
$$

Theorem 1.3 If $n$ is superabundant, then $n$ is an Hardy-Ramanujan integer [2].

Theorem 1.4 The smallest counterexample of the Robin inequality greater than 5040 must be a superabundant number [1].

On the other hand, suppose that $n>5040$ is the possible smallest counterexample of the Robin inequality, then we prove that $q_{m}>e^{30.99733785}, 1<\frac{1.25 \times \log (4.7312714399)}{\log q_{m}}+$ $\frac{\log N_{m}}{\log n},(\log n)^{\beta}<1.0501395952 \times \log \left(N_{m}\right)$ and $n<(4.7312714399)^{m} \times N_{m}$, where $N_{m}=\prod_{i=1}^{m} q_{i}$ is the primorial number of order $m, q_{m}$ is the largest prime divisor of $n$ and $\beta=\prod_{i=1}^{m} \frac{q_{i}^{a_{i}+1}}{q_{i}^{a_{i}+1}-1}$ when $n$ is an Hardy-Ramanujan integer of the form $\prod_{i=1}^{m} q_{i}^{a_{i}}$.

\section{A Central Lemma}

These are known results:

Lemma 2.1 [4]. For $n>1$ :

$$
f(n)<\prod_{q \mid n} \frac{q}{q-1} .
$$


Lemma 2.2 [5].

$$
\prod_{k=1}^{\infty} \frac{1}{1-\frac{1}{q_{k}^{2}}}=\zeta(2)=\frac{\pi^{2}}{6}
$$

The following is a key lemma. It gives an upper bound on $f(n)$ that holds for all natural numbers $n$. The bound is too weak to prove $\operatorname{Robins}(n)$ directly, but is critical because it holds for all natural numbers $n$. Further the bound only uses the primes that divide $n$ and not how many times they divide $n$.

Lemma 2.3 Let $n>1$ and let all its prime divisors be $q_{1}<\cdots<q_{m}$. Then,

$$
f(n)<\frac{\pi^{2}}{6} \times \prod_{i=1}^{m} \frac{q_{i}+1}{q_{i}} .
$$

Proof Putting together the lemmas 2.1 and 2.2 yields the proof:

$$
f(n)<\prod_{i=1}^{m}\left(\frac{q_{i}}{q_{i}-1}\right)=\prod_{i=1}^{m}\left(\frac{q_{i}+1}{q_{i}} \times \frac{1}{1-\frac{1}{q_{i}^{2}}}\right)<\frac{\pi^{2}}{6} \times \prod_{i=1}^{m} \frac{q_{i}+1}{q_{i}} .
$$

\section{Robin on Divisibility}

We know the following lemmas:

Lemma 3.1 [6]. Let $n>e^{e^{23.762143}}$ and let all its prime divisors be $q_{1}<\cdots<q_{m}$, then

$$
\left(\prod_{i=1}^{m} \frac{q_{i}}{q_{i}-1}\right)<\frac{1771561}{1771560} \times e^{\gamma} \times \log \log n .
$$

Lemma 3.2 Robins $(n)$ holds for all natural numbers $10^{10^{13.11485}} \geq n>5040$ [7].

Theorem 3.3 Suppose $n>5040$. If there exists a prime $q \leq 1771559$ with $q \nmid n$, then Robins $(n)$ holds.

Proof We have that $f(n)<\frac{1771561}{1771560} \times e^{\gamma} \times \log \log (n)$ for any number $n>10^{10^{13.11485}}$ since the inequality $10^{10^{13.11485}}>e^{e^{23.762143}}$ is satisfied. Note that $f(n)<\frac{n}{\varphi(n)}=\prod_{q \mid n} \frac{q}{q-1}$ from the lemma 2.1, where $\varphi(x)$ is the Euler's totient function. Suppose that $n$ is not divisible by some prime $q \leq 1771559$ and $n \geq 10^{10^{13.11485}}$. Then,

$$
\begin{aligned}
f(n) & <\frac{n}{\varphi(n)} \\
& =\frac{n \times q}{\varphi(n \times q)} \times \frac{q-1}{q} \\
& <\frac{1771561}{1771560} \times \frac{q-1}{q} \times e^{\gamma} \times \log \log (n \times q)
\end{aligned}
$$


and

$$
\begin{aligned}
\frac{f(n)}{e^{\gamma} \times \log \log (n)} & <\frac{1771561}{1771560} \times \frac{q-1}{q} \times \frac{\log \log (n \times q)}{\log \log (n)} \\
& \leq \frac{1771561}{1771560} \times \frac{q-1}{q} \times \frac{\log \log (n \times q)}{\log \log (n)} \\
& =\frac{1771561}{1771560} \times \frac{q-1}{q} \times \frac{\log \log (n)+\log \left(1+\frac{\log (q)}{\log (n)}\right)}{\log \log (n)} \\
& =\frac{1771561}{1771560} \times \frac{q-1}{q} \times\left(1+\frac{\log \left(1+\frac{\log (q)}{\log (n)}\right)}{\log \log (n)}\right)
\end{aligned}
$$

So

$$
\frac{f(n)}{e^{\gamma} \times \log \log (n)}<\frac{1771561}{1771560} \times \frac{q-1}{q} \times\left(1+\frac{\log \left(1+\frac{\log (q)}{\log (n)}\right)}{\log \log (n)}\right)
$$

for $n \geq 10^{10^{13.11485}}$. The right hand side is less than 1 for $q \leq 1771559$ and $n \geq$ $10^{10^{13.11485}}$. Therefore, Robins $(n)$ holds.

\section{On the Greatest Prime Divisor}

We know that

Lemma 4.1 [9]. For $x \geq 286$ :

$$
\prod_{q \leq x} \frac{q}{q-1}<e^{\gamma} \times\left(\log x+\frac{1}{2 \times \log (x)}\right) .
$$

Theorem 4.2 Let $\prod_{i=1}^{m} q_{i}^{a_{i}}$ be the representation of $n$ as a product of primes $q_{1}<$ $\cdots<q_{m}$ with natural numbers as exponents $a_{1}, \ldots, a_{m}$. If $n>5040$ is the smallest integer such that Robins $(n)$ does not hold, then $q_{m}>e^{30.99733785}$.

Proof According to the theorems 1.3 and 1.4, the primes $q_{1}<\cdots<q_{m}$ must be the first $m$ consecutive primes and $a_{1} \geq a_{2} \geq \cdots \geq a_{m} \geq 0$ since $n>5040$ should be an Hardy-Ramanujan integer. From the theorem 3.3, we know that necessarily $q_{m} \geq 1771559$. So,

$$
e^{\gamma} \times \log \log n \leq f(n)<\prod_{q \leq q_{m}} \frac{q}{q-1}<e^{\gamma} \times\left(\log q_{m}+\frac{1}{2 \times \log \left(q_{m}\right)}\right)
$$

because of the lemmas 2.1 and 4.1. Hence,

$$
\log \log n-\frac{1}{2 \times \log \left(q_{m}\right)}<\log q_{m}
$$


However, from the lemma 3.2 and theorem 3.3, we would obtain that

$$
\begin{aligned}
\log \log n-\frac{1}{2 \times \log \left(q_{m}\right)} & \geq 13.11485 \times \log (10)+\log \log 10-\frac{1}{2 \times \log (1771559)} \\
& >30.99733785
\end{aligned}
$$

Since, we have that

$$
\log q_{m}>\log \log n-\frac{1}{2 \times \log \left(q_{m}\right)}>30.99733785
$$

then, we would obtain that $q_{m}>e^{30.99733785}$ under the assumption that $n>5040$ is the smallest integer such that Robins $(n)$ does not hold.

\section{Some Feasible Cases}

In basic number theory, for a given prime number $p$, the $p$-adic order of a natural number $n$ is the highest exponent $v_{p} \geq 0$ such that $p^{v_{p}}$ divides $n$. This is a known result:

Lemma 5.1 In general, we know that Robins ( $n$ ) holds for a natural number $n>5040$ that satisfies $v_{2}(n) \leq 19$, where $v_{p}(n)$ is the p-adic order of $n$ [6].

We can easily prove that $\operatorname{Robins}(n)$ is true for certain kind of numbers:

Lemma 5.2 Robins $(n)$ holds for $n>5040$ when $q \leq 7$, where $q$ is the largest prime divisor of $n$.

Proof This is an immediate consequence of theorem 3.3.

The next theorem implies that $\operatorname{Robins}(n)$ holds for a wide range of natural numbers $n>5040$.

Theorem 5.3 Let $\frac{\pi^{2}}{6} \times \log \log n^{\prime} \leq \log \log n$ for some $n>5040$ such that $n^{\prime}$ is the square free kernel of the natural number $n$. Then Robins $(n)$ holds.

Proof Let $n^{\prime}$ be the square free kernel of the natural number $n$, that is the product of the distinct primes $q_{1}, \ldots, q_{m}$. By assumption we have that

$$
\frac{\pi^{2}}{6} \times \log \log n^{\prime} \leq \log \log n
$$

For all square free $n^{\prime} \leq 5040$, Robins $\left(n^{\prime}\right)$ holds if and only if $n^{\prime} \notin\{2,3,5,6,10,30\}$ [4]. However, Robins $(n)$ holds for all $n>5040$ when $n^{\prime} \in\{2,3,5,6,10,15,30\}$ due to the lemma 5.2. When $n^{\prime}>5040$, we know that Robins $\left(n^{\prime}\right)$ holds and so

$$
f\left(n^{\prime}\right)<e^{\gamma} \times \log \log n^{\prime}
$$

because of the theorem 1.2. By the previous lemma 2.3:

$$
f(n)<\frac{\pi^{2}}{6} \times \prod_{i=1}^{m} \frac{q_{i}+1}{q_{i}} .
$$


Suppose by way of contradiction that $\operatorname{Robins}(n)$ fails. Then

$$
f(n) \geq e^{\gamma} \times \log \log n \text {. }
$$

We claim that

$$
\frac{\pi^{2}}{6} \times \prod_{i=1}^{m} \frac{q_{i}+1}{q_{i}}>e^{\gamma} \times \log \log n .
$$

Since otherwise we would have a contradiction. This shows that

$$
\frac{\pi^{2}}{6} \times \prod_{i=1}^{m} \frac{q_{i}+1}{q_{i}}>\frac{\pi^{2}}{6} \times e^{\gamma} \times \log \log n^{\prime} .
$$

Thus

$$
\prod_{i=1}^{m} \frac{q_{i}+1}{q_{i}}>e^{\gamma} \times \log \log n^{\prime}
$$

and

$$
\prod_{i=1}^{m} \frac{q_{i}+1}{q_{i}}>f\left(n^{\prime}\right)
$$

This is a contradiction since $f\left(n^{\prime}\right)$ is equal to

$$
\frac{\left(q_{1}+1\right) \times \cdots \times\left(q_{m}+1\right)}{q_{1} \times \cdots \times q_{m}}
$$

according to the formula $f(x)$ for the square free numbers [4].

\section{On Possible Counterexample}

For every prime number $p_{n}>2$, we define the sequence $Y_{n}=\frac{e^{\frac{1}{2 \times \log \left(p_{n}\right)}}}{\left(1-\frac{1}{\log \left(p_{n}\right)}\right)}$.

Lemma 6.1 For every prime number $p_{n}>2$, the sequence $Y_{n}$ is strictly decreasing.

Proof This lemma is obvious.

In mathematics, the Chebyshev function $\theta(x)$ is given by

$$
\theta(x)=\sum_{p \leq x} \log p
$$

where $p \leq x$ means all the prime numbers $p$ that are less than or equal to $x$.

Lemma 6.2 [9]. For $x \geq 41$ :

$$
\theta(x)>\left(1-\frac{1}{\log (x)}\right) \times x .
$$

We will prove another important inequality: 
Lemma 6.3 Let $q_{1}, q_{2}, \ldots, q_{m}$ denote the first $m$ consecutive primes such that $q_{1}<$ $q_{2}<\cdots<q_{m}$ and $q_{m}>286$. Then

$$
\prod_{i=1}^{m} \frac{q_{i}}{q_{i}-1}<e^{\gamma} \times \log \left(Y_{m} \times \theta\left(q_{m}\right)\right) .
$$

Proof From the lemma 6.2, we know that

$$
\theta\left(q_{m}\right)>\left(1-\frac{1}{\log \left(q_{m}\right)}\right) \times q_{m}
$$

In this way, we can show that

$$
\begin{aligned}
\log \left(Y_{m} \times \theta\left(q_{m}\right)\right) & >\log \left(Y_{m} \times\left(1-\frac{1}{\log \left(q_{m}\right)}\right) \times q_{m}\right) \\
& =\log q_{m}+\log \left(Y_{m} \times\left(1-\frac{1}{\log \left(q_{m}\right)}\right)\right) .
\end{aligned}
$$

We know that

$$
\begin{aligned}
\log \left(Y_{m} \times\left(1-\frac{1}{\log \left(q_{m}\right)}\right)\right) & =\log \left(\frac{e^{\frac{1}{2 \times \log \left(q_{m}\right)}}}{\left(1-\frac{1}{\log \left(q_{m}\right)}\right)} \times\left(1-\frac{1}{\log \left(q_{m}\right)}\right)\right) \\
& =\log \left(e^{\frac{1}{2 \times \log \left(q_{m}\right)}}\right) \\
& =\frac{1}{2 \times \log \left(q_{m}\right)} .
\end{aligned}
$$

Consequently, we obtain that

$$
\log q_{m}+\log \left(Y_{m} \times\left(1-\frac{1}{\log \left(q_{m}\right)}\right)\right) \geq\left(\log q_{m}+\frac{1}{2 \times \log \left(q_{m}\right)}\right) .
$$

Due to the lemma 4.1, we prove that

$$
\prod_{i=1}^{m} \frac{q_{i}}{q_{i}-1}<e^{\gamma} \times\left(\log q_{m}+\frac{1}{2 \times \log \left(q_{m}\right)}\right)<e^{\gamma} \times \log \left(Y_{m} \times \theta\left(q_{m}\right)\right)
$$

when $q_{m}>286$.

We use the following lemma:

Lemma 6.4 [6]. Let $\prod_{i=1}^{m} q_{i}^{a_{i}}$ be the representation of $n$ as a product of primes $q_{1}<$ $\cdots<q_{m}$ with natural numbers as exponents $a_{1}, \ldots, a_{m}$. Then,

$$
f(n)=\left(\prod_{i=1}^{m} \frac{q_{i}}{q_{i}-1}\right) \times \prod_{i=1}^{m}\left(1-\frac{1}{q_{i}^{a_{i}+1}}\right) .
$$

The following theorems have a great significance, because these mean that the possible smallest counterexample of the Robin inequality greater than 5040 must be very close to its square free kernel. 
Theorem 6.5 Let $\prod_{i=1}^{m} q_{i}^{a_{i}}$ be the representation of $n$ as a product of primes $q_{1}<$ $\cdots<q_{m}$ with natural numbers as exponents $a_{1}, \ldots, a_{m}$. If $n>5040$ is the smallest integer such that Robins $(n)$ does not hold, then $(\log n)^{\beta}<Y_{m} \times \log \left(N_{m}\right)$, where $N_{m}=$ $\prod_{i=1}^{m} q_{i}$ is the primorial number of order $m$ and $\beta=\prod_{i=1}^{m} \frac{q_{i}^{a_{i}+1}}{q_{i}^{a_{i}+1}-1}$.

Proof According to the theorems 1.3 and 1.4, the primes $q_{1}<\cdots<q_{m}$ must be the first $m$ consecutive primes and $a_{1} \geq a_{2} \geq \cdots \geq a_{m} \geq 0$ since $n>5040$ should be an Hardy-Ramanujan integer. From the theorem 4.2, we know that necessarily $q_{m}>e^{30.99733785}$. From the lemma 6.4, we note that

$$
f(n)=\left(\prod_{i=1}^{m} \frac{q_{i}}{q_{i}-1}\right) \times \prod_{i=1}^{m}\left(1-\frac{1}{q_{i}^{a_{i}+1}}\right) .
$$

However, we know that

$$
\prod_{i=1}^{m} \frac{q_{i}}{q_{i}-1}<e^{\gamma} \times \log \left(Y_{m} \times \log \left(N_{m}\right)\right)
$$

because of the lemma 6.3 when $q_{m}>e^{30.99733785}$. If we multiply by $\prod_{i=1}^{m}\left(1-\frac{1}{q_{i}^{a_{i}+1}}\right)$ the both sides of the previous inequality, then we obtain that

$$
f(n)<e^{\gamma} \times \log \left(Y_{m} \times \log \left(N_{m}\right)\right) \times \prod_{i=1}^{m}\left(1-\frac{1}{q_{i}^{a_{i}+1}}\right) .
$$

If $n$ is the smallest integer exceeding 5040 that does not satisfy the Robin inequality, then

$$
e^{\gamma} \times \log \log n<e^{\gamma} \times \log \left(Y_{m} \times \log \left(N_{m}\right)\right) \times \prod_{i=1}^{m}\left(1-\frac{1}{q_{i}^{a_{i}+1}}\right)
$$

because of

$$
e^{\gamma} \times \log \log n \leq f(n)
$$

That is the same as

$$
\prod_{i=1}^{m} \frac{q_{i}^{a_{i}+1}}{q_{i}^{a_{i}+1}-1} \times \log \log n<\log \left(Y_{m} \times \log \left(N_{m}\right)\right)
$$

which is equivalent to

$$
(\log n)^{\beta}<Y_{m} \times \log \left(N_{m}\right)
$$

where $\beta=\prod_{i=1}^{m} \frac{q_{i}^{a_{i}+1}}{q_{i}^{a_{i}+1}-1}$. Therefore, the proof is done.

Theorem 6.6 Let $\prod_{i=1}^{m} q_{i}^{a_{i}}$ be the representation of $n$ as a product of primes $q_{1}<$ $\cdots<q_{m}$ with natural numbers as exponents $a_{1}, \ldots, a_{m}$. If $n>5040$ is the smallest integer such that Robins $(n)$ does not hold, then $(\log n)^{\beta}<1.0501395952 \times \log \left(N_{m}\right)$, where $N_{m}=\prod_{i=1}^{m} q_{i}$ is the primorial number of order $m$ and $\beta=\prod_{i=1}^{m} \frac{q_{i}^{a_{i}+1}}{q_{i}^{a_{i}+1}-1}$. 
Proof From the theorem 4.2, we know that necessarily $q_{m}>e^{30.99733785}$. Using the theorem 6.5 , we obtain that

$$
(\log n)^{\beta}<1.0501395952 \times \log \left(N_{m}\right)
$$

due to the lemma 6.1 since we have that $Y_{m}<1.0501395952$ when $q_{m}>e^{30.99733785}$.

Theorem 6.7 Let $\prod_{i=1}^{m} q_{i}^{a_{i}}$ be the representation of $n$ as a product of primes $q_{1}<$ $\cdots<q_{m}$ with natural numbers as exponents $a_{1}, \ldots, a_{m}$. If $n>5040$ is the smallest integer such that Robins $(n)$ does not hold, then $n<(4.7312714399)^{m} \times N_{m}$, where $N_{m}=\prod_{i=1}^{m} q_{i}$ is the primorial number of order $m$.

Proof According to the theorems 1.3 and 1.4, the primes $q_{1}<\cdots<q_{m}$ must be the first $m$ consecutive primes and $a_{1} \geq a_{2} \geq \cdots \geq a_{m} \geq 0$ since $n>5040$ should be an Hardy-Ramanujan integer. From the lemma 6.3, we know that

$$
\prod_{i=1}^{m} \frac{q_{i}}{q_{i}-1}<e^{\gamma} \times \log \left(Y_{m} \times \theta\left(q_{m}\right)\right)=e^{\gamma} \times \log \log \left(N_{m}^{Y_{m}}\right)
$$

for $q_{m}>e^{30.99733785}$. In this way, if $n>5040$ is the smallest integer such that Robins $(n)$ does not hold, then $n<N_{m}^{Y_{m}}$ since by the lemma 2.1 we have that

$$
f(n)<\prod_{i=1}^{m} \frac{q_{i}}{q_{i}-1} .
$$

That is the same as $n<N_{m}^{Y_{m}-1} \times N_{m}$. We can check that $q_{m}^{Y_{m}-1}$ is monotonically decreasing for all primes $q_{m}>e^{30.99733785}$. Certainly, the function

$$
g(x)=x^{\left(\frac{e^{\frac{1}{2 \times \log (x)}}}{\left(1-\frac{1}{\log (x)}\right)}-1\right)}
$$

complies that its derivative is lesser than zero for all real numbers $x \geq e^{30.99733785}$. Consequently, we would have that

$$
q_{m}^{Y_{m}-1}<g\left(e^{30.99733785}\right)<4.7312714399
$$

for all primes $q_{m}>e^{30.99733785}$. Moreover, we would obtain that

$$
q_{m}^{Y_{m}-1}>q_{j}^{Y_{m}-1}
$$

for every integer $1 \leq j<m$. Finally, we can state that $n<(4.7312714399)^{m} \times N_{m}$ since $N_{m}^{Y_{m}-1}<(4.7312714399)^{m}$ when $n>5040$ is the smallest integer such that $\operatorname{Robins}(n)$ does not hold.

We know the following results:

Lemma 6.8 [9]. For $x \geq 114$ :

$$
\pi(x)<1.25 \times \frac{x}{\log x}
$$

where $\pi(x)$ is the prime counting function. 
Lemma 6.9 If $n>5040$ is the smallest integer such that Robins( $n)$ does not hold, then $p<\log n$ where $p$ is the largest prime divisor of $n$ [4].

Theorem 6.10 Let $\prod_{i=1}^{m} q_{i}^{a_{i}}$ be the representation of $n$ as a product of primes $q_{1}<$ $\cdots<q_{m}$ with natural numbers as exponents $a_{1}, \ldots, a_{m}$. If $n>5040$ is the smallest integer such that Robins $(n)$ does not hold, then $1<\frac{1.25 \times \log (4.7312714399)}{\log q_{m}}+\frac{\log N_{m}}{\log n}$, where $N_{m}=\prod_{i=1}^{m} q_{i}$ is the primorial number of order $m$.

Proof Note that $n<(4.7312714399)^{m} \times N_{m}$ when $n$ is the smallest integer such that Robins $(n)$ does not hold. If we apply the logarithm to the both sides, then

$$
\log n<\log (4.7312714399)^{m}+\log N_{m}
$$

which is equivalent to

$$
\log n<m \times \log (4.7312714399)+\log N_{m} .
$$

According to the lemma 6.8, we have that

$$
\log n<1.25 \times \frac{q_{m}}{\log q_{m}} \times \log (4.7312714399)+\log N_{m} .
$$

From the lemma 6.9, we would have

$$
\log n<1.25 \times \frac{\log n}{\log q_{m}} \times \log (4.7312714399)+\log N_{m} .
$$

which is the same as

$$
1<\frac{1.25 \times \log (4.7312714399)}{\log q_{m}}+\frac{\log N_{m}}{\log n}
$$

after of dividing by $\log n$.

\section{Another Bound}

This is a known result:

Lemma 7.1 [9]. For $x>1$ :

$$
\sum_{q \leq x} \frac{1}{q}<\log \log x+B+\frac{1}{\log ^{2} x}
$$

where

$$
B=0.2614972128 \cdots
$$

denotes the (Meissel-)Mertens constant [9].

We show another result:

Lemma 7.2 For $x \geq 11$, we have

$$
\sum_{q \leq x} \frac{1}{q}<\log \log x+\gamma-0.12 .
$$


Proof Let's define $H=\gamma-B$. The lemma 7.1 is the same as

$$
\sum_{q \leq x} \frac{1}{q}<\log \log x+\gamma-\left(H-\frac{1}{\log ^{2} x}\right) .
$$

For $x \geq 11$,

$$
\left(H-\frac{1}{\log ^{2} x}\right)>\left(0.31-\frac{1}{\log ^{2} 11}\right)>0.12
$$

and thus,

$$
\sum_{q \leq x} \frac{1}{q}<\log \log x+\gamma-\left(H-\frac{1}{\log ^{2} x}\right)<\log \log x+\gamma-0.12
$$

\section{On a Square Free Number}

We know the following results:

Lemma 8.1 [4]. For $0<a<b$ :

$$
\frac{\log b-\log a}{b-a}=\frac{1}{(b-a)} \int_{a}^{b} \frac{d t}{t}>\frac{1}{b} .
$$

Lemma 8.2 [4]. For $q>0$ :

$$
\log (q+1)-\log q=\int_{q}^{q+1} \frac{d t}{t}<\frac{1}{q} .
$$

We know from the theorem 1.2 that $\operatorname{Robins}(n)$ holds for all natural numbers $n>$ 5040 that are square free.

Lemma 8.3 For a square free number

$$
n=q_{1} \times \cdots \times q_{m}
$$

such that $q_{1}<q_{2}<\cdots<q_{m}$ are odd prime numbers, $q_{m} \geq 11$ and $3 \nmid n$, then:

$$
\frac{\pi^{2}}{6} \times \frac{3}{2} \times \sigma(n) \leq e^{\gamma} \times n \times \log \log \left(2^{19} \times n\right) .
$$

Proof By induction with respect to $\omega(n)$, that is the number of distinct prime factors of $n$ [4]. Put $\omega(n)=m$ [4]. We need to prove the assertion for those integers with $m=1$. From a square free number $n$, we obtain

$$
\sigma(n)=\left(q_{1}+1\right) \times\left(q_{2}+1\right) \times \cdots \times\left(q_{m}+1\right)
$$

when $n=q_{1} \times q_{2} \times \cdots \times q_{m}$ [4]. In this way, for every prime number $q_{i} \geq 11$, then we need to prove

$$
\frac{\pi^{2}}{6} \times \frac{3}{2} \times\left(1+\frac{1}{q_{i}}\right) \leq e^{\gamma} \times \log \log \left(2^{19} \times q_{i}\right) .
$$


For $q_{i}=11$, we have

$$
\frac{\pi^{2}}{6} \times \frac{3}{2} \times\left(1+\frac{1}{11}\right) \leq e^{\gamma} \times \log \log \left(2^{19} \times 11\right)
$$

is actually true. For another prime number $q_{i}>11$, we have

$$
\left(1+\frac{1}{q_{i}}\right)<\left(1+\frac{1}{11}\right)
$$

and

$$
\log \log \left(2^{19} \times 11\right)<\log \log \left(2^{19} \times q_{i}\right)
$$

which clearly implies that the inequality (8.4) is true for every prime number $q_{i} \geq 11$. Now, suppose it is true for $m-1$, with $m \geq 2$ and let us consider the assertion for those square free $n$ with $\omega(n)=m$ [4]. So let $n=q_{1} \times \cdots \times q_{m}$ be a square free number and assume that $q_{1}<\cdots<q_{m}$ for $q_{m} \geq 11$.

Case 1: $q_{m} \geq \log \left(2^{19} \times q_{1} \times \cdots \times q_{m-1} \times q_{m}\right)=\log \left(2^{19} \times n\right)$.

By the induction hypothesis we have

$\frac{\pi^{2}}{6} \times \frac{3}{2} \times\left(q_{1}+1\right) \times \cdots \times\left(q_{m-1}+1\right) \leq e^{\gamma} \times q_{1} \times \cdots \times q_{m-1} \times \log \log \left(2^{19} \times q_{1} \times \cdots \times q_{m-1}\right)$

and hence

$$
\begin{gathered}
\frac{\pi^{2}}{6} \times \frac{3}{2} \times\left(q_{1}+1\right) \times \cdots \times\left(q_{m-1}+1\right) \times\left(q_{m}+1\right) \leq \\
e^{\gamma} \times q_{1} \times \cdots \times q_{m-1} \times\left(q_{m}+1\right) \times \log \log \left(2^{19} \times q_{1} \times \cdots \times q_{m-1}\right)
\end{gathered}
$$

when we multiply the both sides of the inequality by $\left(q_{m}+1\right)$. We want to show

$$
\begin{gathered}
e^{\gamma} \times q_{1} \times \cdots \times q_{m-1} \times\left(q_{m}+1\right) \times \log \log \left(2^{19} \times q_{1} \times \cdots \times q_{m-1}\right) \leq \\
e^{\gamma} \times q_{1} \times \cdots \times q_{m-1} \times q_{m} \times \log \log \left(2^{19} \times q_{1} \times \cdots \times q_{m-1} \times q_{m}\right)=e^{\gamma} \times n \times \log \log \left(2^{19} \times n\right) .
\end{gathered}
$$

Indeed the previous inequality is equivalent with

$q_{m} \times \log \log \left(2^{19} \times q_{1} \times \cdots \times q_{m-1} \times q_{m}\right) \geq\left(q_{m}+1\right) \times \log \log \left(2^{19} \times q_{1} \times \cdots \times q_{m-1}\right)$

or alternatively

$$
\begin{gathered}
\frac{q_{m} \times\left(\log \log \left(2^{19} \times q_{1} \times \cdots \times q_{m-1} \times q_{m}\right)-\log \log \left(2^{19} \times q_{1} \times \cdots \times q_{m-1}\right)\right)}{\log q_{m}} \geq \\
\frac{\log \log \left(2^{19} \times q_{1} \times \cdots \times q_{m-1}\right)}{\log q_{m}} .
\end{gathered}
$$

We can apply the inequality in lemma 8.1 just using $b=\log \left(2^{19} \times q_{1} \times \cdots \times q_{m-1} \times\right.$ $\left.q_{m}\right)$ and $a=\log \left(2^{19} \times q_{1} \times \cdots \times q_{m-1}\right)$. Certainly, we have

$$
\begin{gathered}
\log \left(2^{19} \times q_{1} \times \cdots \times q_{m-1} \times q_{m}\right)-\log \left(2^{19} \times q_{1} \times \cdots \times q_{m-1}\right)= \\
\log \frac{2^{19} \times q_{1} \times \cdots \times q_{m-1} \times q_{m}}{2^{19} \times q_{1} \times \cdots \times q_{m-1}}=\log q_{m} .
\end{gathered}
$$


In this way, we obtain

$$
\begin{gathered}
\frac{q_{m} \times\left(\log \log \left(2^{19} \times q_{1} \times \cdots \times q_{m-1} \times q_{m}\right)-\log \log \left(2^{19} \times q_{1} \times \cdots \times q_{m-1}\right)\right)}{\log q_{m}}> \\
\frac{q_{m}}{\log \left(2^{19} \times q_{1} \times \cdots \times q_{m}\right)} .
\end{gathered}
$$

Using this result we infer that the original inequality is certainly satisfied if the next inequality is satisfied

$$
\frac{q_{m}}{\log \left(2^{19} \times q_{1} \times \cdots \times q_{m}\right)} \geq \frac{\log \log \left(2^{19} \times q_{1} \times \cdots \times q_{m-1}\right)}{\log q_{m}}
$$

which is trivially true for $q_{m} \geq \log \left(2^{19} \times q_{1} \times \cdots \times q_{m-1} \times q_{m}\right)$ [4].

Case 2: $q_{m}<\log \left(2^{19} \times q_{1} \times \cdots \times q_{m-1} \times q_{m}\right)=\log \left(2^{19} \times n\right)$.

We need to prove

$$
\frac{\pi^{2}}{6} \times \frac{3}{2} \times \frac{\sigma(n)}{n} \leq e^{\gamma} \times \log \log \left(2^{19} \times n\right)
$$

We know $\frac{3}{2}<1.503<\frac{4}{2.66}$. Nevertheless, we could have

$$
\frac{3}{2} \times \frac{\sigma(n)}{n} \times \frac{\pi^{2}}{6}<\frac{4 \times \sigma(n)}{3 \times n} \times \frac{\pi^{2}}{2 \times 2.66}
$$

and therefore, we only need to prove

$$
\frac{\sigma(3 \times n)}{3 \times n} \times \frac{\pi^{2}}{5.32} \leq e^{\gamma} \times \log \log \left(2^{19} \times n\right)
$$

where this is possible because of $3 \nmid n$. If we apply the logarithm to the both sides of the inequality, then we obtain

$\log \left(\frac{\pi^{2}}{5.32}\right)+(\log (3+1)-\log 3)+\sum_{i=1}^{m}\left(\log \left(q_{i}+1\right)-\log q_{i}\right) \leq \gamma+\log \log \log \left(2^{19} \times n\right)$.

In addition, note that $\log \left(\frac{\pi^{2}}{5.32}\right)<\frac{1}{2}+0.12$. However, we know

$$
\gamma+\log \log q_{m}<\gamma+\log \log \log \left(2^{19} \times n\right)
$$

since $q_{m}<\log \left(2^{19} \times n\right)$. We use that lemma 8.2 for each term $\log (q+1)-\log q$ and thus,

$$
0.12+\frac{1}{2}+\frac{1}{3}+\frac{1}{q_{1}}+\cdots+\frac{1}{q_{m}} \leq 0.12+\sum_{q \leq q_{m}} \frac{1}{q} \leq \gamma+\log \log q_{m}
$$

where $q_{m} \geq 11$. Hence, it is enough to prove

$$
\sum_{q \leq q_{m}} \frac{1}{q} \leq \gamma+\log \log q_{m}-0.12
$$

but this is true according to the lemma 7.2 for $q_{m} \geq 11$. In this way, we finally show the lemma is indeed satisfied. 


\section{Main Insight}

The next result is a main insight.

Theorem 9.1 Let $n>5040$ and let all its prime divisors be $q_{1}<\cdots<q_{m}$. When $q_{m} \geq 11,3 \nmid n$ and $2^{20} \mid n$, then

$$
\frac{\pi^{2}}{6} \times \prod_{i=1}^{m} \frac{q_{i}+1}{q_{i}} \leq e^{\gamma} \times \log \log n .
$$

Proof We need to prove that

$$
\frac{\pi^{2}}{6} \times \prod_{i=1}^{m} \frac{q_{i}+1}{q_{i}} \leq e^{\gamma} \times \log \log n .
$$

Using the formula (8.3) for the square free numbers, then we obtain that is equivalent to

$$
\frac{\pi^{2}}{6} \times \frac{\sigma\left(n^{\prime}\right)}{n^{\prime}} \leq e^{\gamma} \times \log \log n
$$

where $n^{\prime}=q_{1} \times \cdots \times q_{m}$ is the square free kernel of the natural number $n$ [4]. We know that $2^{20} \mid n$ and thus,

$$
e^{\gamma} \times n^{\prime} \times \log \log \left(2^{19} \times \frac{n^{\prime}}{2}\right) \leq e^{\gamma} \times n^{\prime} \times \log \log n
$$

because of $2^{19} \times \frac{n^{\prime}}{2} \leq n$ where $2^{20} \mid n$ and $2 \mid n^{\prime}$. So,

$$
\frac{\pi^{2}}{6} \times \sigma\left(n^{\prime}\right) \leq e^{\gamma} \times n^{\prime} \times \log \log \left(2^{19} \times \frac{n^{\prime}}{2}\right) .
$$

According to the formula (8.3) for the square free numbers and $2 \mid n^{\prime}$, then,

$$
\frac{\pi^{2}}{6} \times 3 \times \sigma\left(\frac{n^{\prime}}{2}\right) \leq e^{\gamma} \times 2 \times \frac{n^{\prime}}{2} \times \log \log \left(2^{19} \times \frac{n^{\prime}}{2}\right)
$$

which is the same as

$$
\frac{\pi^{2}}{6} \times \frac{3}{2} \times \sigma\left(\frac{n^{\prime}}{2}\right) \leq e^{\gamma} \times \frac{n^{\prime}}{2} \times \log \log \left(2^{19} \times \frac{n^{\prime}}{2}\right)
$$

where this is true according to the lemma 8.3 when $3 \nmid \frac{n^{\prime}}{2}$ and $q_{m} \geq 11$. To sum up, the proof is complete.

\section{Acknowledgments}

The author would like to thank Richard J. Lipton and Craig Helfgott for helpful comments and his mother, maternal brother and his friend Sonia for their support. 


\section{References}

1. Akbary, A., Friggstad, Z.: Superabundant numbers and the Riemann hypothesis. The American Mathematical Monthly 116(3), 273-275 (2009). DOI doi:10.4169/193009709X470128

2. Alaoglu, L., Erdős, P.: On highly composite and similar numbers. Transactions of the American Mathematical Society 56(3), 448-469 (1944). DOI doi:10.2307/1990319

3. Borwein, P.B., Choi, S., Rooney, B., Weirathmueller, A.: The Riemann Hypothesis: A Resource for the Afficionado and Virtuoso Alike, vol. 27. Springer Science \& Business Media (2008)

4. Choie, Y., Lichiardopol, N., Moree, P., Solé, P.: On Robin's criterion for the Riemann hypothesis. Journal de Théorie des Nombres de Bordeaux 19(2), 357-372 (2007). DOI doi:10.5802/jtnb.591

5. Edwards, H.M.: Riemann's Zeta Function. Dover Publications (2001)

6. Hertlein, A.: Robin's Inequality for New Families of Integers. Integers 18 (2018)

7. Platt, D.J., Morrill, T.: Robin's inequality for 20-free integers. INTEGERS: Electronic Journal of Combinatorial Number Theory (2021)

8. Robin, G.: Grandes valeurs de la fonction somme des diviseurs et hypothèse de Riemann. J. Math. pures appl 63(2), 187-213 (1984)

9. Rosser, J.B., Schoenfeld, L.: Approximate Formulas for Some Functions of Prime Numbers. Illinois Journal of Mathematics 6(1), 64-94 (1962). DOI doi:10.1215/ijm/1255631807 